(Wheeler), and a new theory of relativistic compact objects (Hoyle).

Observational cosmology and galaxy evolution are discussed in the fourth section which reviews the age of galaxies and globular clusters with a view to finding the Hubble constant and deceleration parameter (Sandage), the evolution of radio sources (Rees), the curious mystery of $\log N-\log S$ (Hoyle), the $\mathrm{X}$-ray background radiation (Friedman), and the $X$-ray and microwave background (Morrison).

The final section contains excellent summaries of the observational (E. M. Burbidge) and theoretical (L. Woltjer) positions, a list of observational and theoretical conclusions and useful suggestions for future work, and name and subject indices. The total ratio of observation to theory is about 3 to 1 . There is also a reasonable amount of recorded discussion (about one-fifth of the book) of the papers. The quality of editing is high, although there are rare slips as when a discussion refers to previous comments or diagrams which are not recorded.

The lists of agreed conclusions resemble some of the diplomatic communiqués which emerge after heads of state meet in the countryside. The general style of the observational conclusion has the form: There is strong (increasing, extensive) evidence that ... On the other hand, the theoretical conclusions leave the impression . . . It might be possible that . . Most of the theoretical conclusions are really questions, and this indicates the present uncertainty surrounding the foundations of the subject.

Indeed, in his summary, Woltjer remarks: "Even though no strong controversy has developed at this conference it is very clear that, if we try to write down a set of definite conclusions from the theoretical point of view, many of our old disagreements will come to the surface very quickly. This meeting has been extremely successful in part because some of our differences have been set aside for the moment. ..." The more conventional side of this controversy believes that the nuclei have formed by contracting from a more diffuse state and that the observations can be explained by conventional physics and standard general relativity. The alternative view is that the nuclei preceded and produced their less dense surroundings, and new laws of physics are required.

At present, it is much a matter of personal judgment whether one believes conventional physics can explain these discoveries. Do we need new laws of the redshift, new methods of releasing vast amounts of energy? Or are we just too unimaginative in applying existing theory? These questions are so difficult because the observations only supply very limited information about dozens of interacting physical processes. After observing extragalactic phenomena for many decades, it is amazing that we know so little. Yet it is even more amazing that we know so much.

William C. Saslaw

\section{Background to Plasmas}

Sources of Plasma Physics. Vol. 1. By H. S. Green and R. B. Leipnik. Pp. 630. (Wolters-Noordhoff: Groningen, 1970.) 118 Dfl. ; £3.75; \$35.

THE primary object of this work, according to the authors, is to bring together in a single volume discussions of the mathematical foundations of statistical mechanics, the principles of electromagnetism, fluid dynamics and the kinetic theory of gases in order that a second (future) volume on plasma physics may be properly understood. Even in a book of this size, such an ambitious project must of necessity leave out substantial aspects of each of these subjects, particularly when more than a quarter of the available space is devoted to such diverse "mathematical method" topics as measure and probability theory, vector spaces, Fourier analysis, vector analysis, differential equations, calculus of variations and thermodynamics. Most university undergraduates and research workers have orcasion to study these topics in depth before approaching the more formidable fields of magnetohydrodynamics and plasma dynamics. It is very difficult, if not impossible, to give a comprehensive account of all the relevant techniques of mathematical physics in one book. Certainly an extensive knowledge of pure mathematics is required before the relevance of the highly condensed appendices at the end of this book can be appreciated.

Before studying plasmas, almost all students will have attended substantial courses on electromagnetism and relativity, quantum mechanics, hydrodynamics and fluid mechanics. Furthermore, in Europe, such courses will have been given using SI units, whereas this book is written in CGS units and as such will have little appeal to nonmathematicians. After a prolonged struggle to master the SI system, I have no nostalgic longing for Gaussian or other discarded units and I find this return to the past, presumably intended for the benefit of American readers, annoying and confusing, particularly because the book is aimed at students of physics and engineering.

Many of the more difficult problems, for example those on drift analysis, and the publications of Russian authors receive little prominence but perhaps this will be resolved in later volumes. In a work of this kind a fairly detailed historical account is always of value. In this field there is much to be learnt from the lessons and mistakes of the past. For example, has any research worker in magnetohydrodynamics failed to rediscover Ferraro's law of isorotation? Here the historical aspect is played down somewhat and is replaced by concise discussions of the fundamental physical aspects and formulation of the associated mathematical problems.

This emphasis on physics, however, does not permeate throughout the whole book. The mathematical treatment of many problems is very sound but physical interpretations of mathematical results are very brief or non-existent.

It will be interesting to see how a prospective research student, in his first year after graduation, copes with this volume. It is always difficult to assess parts of a multivolume work in isolation, and so after the appearance of the second volume, which deals with a theoretical formulation of plasma physics, it will be possible to give a more precise estimate of this book.

\section{Plumpton}

\section{Molecular Spectra}

The Spectra and Structures of Simple Free Radicals: an Introduction to Molecular Spectroscopy. By Gerhard Herzberg. (The George Fisher-Baker Non-Resident Lectureship in Chemistry at Cornell University.) Pp. xi 226. (Cornell: Ithaca and London, 1971.) $£ 5.25$.

IN the preface to this book the author states that for forty years he had wanted to write a small book of some 200 pages dealing with molecular spectra and structure. However, as all spectroscopists know, during this period he did write a magnificent three-volume monograph of 2,000 pages. Now, at last, he has fulfilled his early ambition, and provided us with a short account of molecular spectra and structure. The role of free radicals is shown by the fact that wherever possible he has chosen the spectra of radicals rather than of stable molecules to illustrate his argument. The result is that we have an effectively complete account of the spectra of free radicals.

This is as it should be. For no one has contributed more than Herzberg to the understanding of these systems; and in many situations, on account of their very short lifetime which prevents any normal study by conventional chemical methods, this is the only way of studying their geometrical structure. How very right it is that his influence in this field should now have been recognized by the award of a Nobel Prize.

It is a strange story that we read. Thus the first chemical detection of polyatomic free radicals, for example 